\title{
Some Conformal Invariants from the Noncommutative Residue for Manifolds with Boundary ${ }^{\star}$
}

\author{
William J. UGALDE
}

Escuela de Matemática, Universidad de Costa Rica, Código postal 2060 San José, Costa Rica E-mail: william.ugalde@ucr.ac.cr

URL: http: //www2.emate.ucr.ac.cr/ ugalde/

Received August 06, 2007, in final form October 31, 2007; Published online November 07, 2007

Original article is available at http://www.emis.de/journals/SIGMA/2007/104/

\begin{abstract}
We review previous work of Alain Connes, and its extension by the author, on some conformal invariants obtained from the noncommutative residue on even dimensional compact manifolds without boundary. Inspired by recent work of Yong Wang, we also address possible generalizations of these conformal invariants to the setting of compact manifolds with boundary.
\end{abstract}

Key words: manifolds with boundary; noncommutative residue; Fredholm module; conformal invariants

2000 Mathematics Subject Classification: 53A30

\section{Introduction}

There is one particular aspect of noncommutative geometry that has historically received less attention than other of its subjects; the use of its machinery to obtain conformal invariants (associated to the underlying manifold). The motivating example in this venue, is a conformal invariant in dimension 4 (Connes [5]) and its extension to higher order even dimensional manifolds by the author [19]. The main idea lies on Theorem IV.4.2.c of Connes [6]. This theorem states that the oriented conformal structure of a compact even-dimensional smooth manifold is uniquely determined by the Fredholm module $(\mathcal{H}, F, \gamma)$ of Connes, Sullivan and Teleman [7]; via the noncommutative residue Res of Adler, Manin, Guillemin, and Wodzicki [1, 13, 12, 23].

In Section 2 of [5] Connes uses his quantized calculus to find a conformal invariant in the 4-dimensional case. A central part of the explicit computation of this conformal invariant is the study of a trilinear functional on smooth functions over the manifold $M^{4}$ given by the relation

$$
\tau\left(f_{0}, f_{1}, f_{2}\right)=\operatorname{Res}\left(f_{0}\left[F, f_{1}\right]\left[F, f_{2}\right]\right) .
$$

Here $F$ is a pseudodifferential operator of order 0 acting on 2 -forms over $M^{4}$. This conformal invariant computed by Connes in the 4-dimensional case is a natural bilinear differential functional of order 4 acting on $C^{\infty}\left(M^{4}\right)$. In [5] it is denoted $\Omega$ and in these notes it is denoted $B_{4} d x$.

This bilinear functional is symmetric, $B_{4}\left(f_{1}, f_{2}\right)=B_{4}\left(f_{2}, f_{1}\right)$, and conformally invariant, in the sense that $\widehat{B_{4}}\left(f_{1}, f_{2}\right)=e^{-4 \eta} B_{4}\left(f_{1}, f_{2}\right)$ for a conformal change of the metric $\widehat{g}=e^{2 \eta} g$. It is also uniquely determined by the relation:

$$
\tau\left(f_{0}, f_{1}, f_{2}\right)=\int_{M} f_{0} B_{4}\left(f_{1}, f_{2}\right) d x, \quad \forall f_{i} \in C^{\infty}\left(M^{4}\right) .
$$

${ }^{\star}$ This paper is a contribution to the Proceedings of the 2007 Midwest Geometry Conference in honor of Thomas P. Branson. The full collection is available at http://www.emis.de/journals/SIGMA/MGC2007.html 
Furthermore, in the 4-dimensional case, Connes has also shown that the Paneitz operator [14] (critical GJMS for $n=4[10]$ ), can be derived from $B_{4}$ by the relation

$$
\int_{M} B_{4}\left(f_{1}, f_{2}\right) d x=\frac{1}{2} \int_{M} f_{1} P_{4}\left(f_{2}\right) d x
$$

Aiming to extend the work of Connes to even dimensional manifolds, in [18] we have proved the following two results:

Theorem 1 of [18]. Let $M$ be an n-dimensional compact conformal manifold without boundary. Let $S$ be a pseudodifferential operator of order 0 acting on sections of a vector bundle over $M$ such that $S^{2} f_{1}=f_{1} S^{2}$ and the pseudodifferential operator $P=\left[S, f_{1}\right]\left[S, f_{2}\right]$ is conformally invariant for any $f_{i} \in C^{\infty}(M)$. Then there exists a unique, symmetric, bilinear, differential functional $B_{n, S}$ of order $n$ conformally invariant in the sense that $\widehat{B_{n, S}}\left(f_{1}, f_{2}\right)=e^{-n \eta} B_{n, S}\left(f_{1}, f_{2}\right)$, for $\widehat{g}=e^{2 \eta} g$, and such that

$$
\operatorname{Res}\left(f_{0}\left[S, f_{1}\right]\left[S, f_{2}\right]\right)=\int_{M} f_{0} B_{n, S}\left(f_{1}, f_{2}\right) d x
$$

for all $f_{i} \in C^{\infty}(M)$.

A particular case of the above result occurs when one works with even-dimensional manifolds for then, it makes sense to consider the Fredholm module $(\mathcal{H}, F)$ associated to $M$. The operator $F$ has the property $F^{2}=1$ and in general $[F, f] \neq 0$ for $f \in C^{\infty}(M)$. Taking $S$ as $F$ in the previous theorem one has:

Theorem 2 of [18]. Let $M$ be a compact conformal manifold without boundary of even dimension $n$ and let $(\mathcal{H}, F)$ be the Fredholm module associated to $M$ by A. Connes [5]. Then, by taking $S=F$ in Theorem 1 of [18] there is a unique, symmetric, and conformally invariant $n$-differential form $B_{n}=B_{n, F}$ such that

$$
\operatorname{Res}\left(f_{0}\left[F, f_{1}\right]\left[F, f_{2}\right]\right)=\int_{M} f_{0} B_{n}\left(f_{1}, f_{2}\right) d x
$$

for all $f_{i} \in C^{\infty}(M)$.

These results are based on the study of the formula for the total symbol $\sigma\left(P_{1} P_{2}\right)$ of the product of two pseudodifferential operators, in the particular case in which one of them is a multiplication operator. The following is the main result of [19]:

Theorem 1. Let $M$ be a compact conformal manifold without boundary of even dimension $n$ and let $(\mathcal{H}, F)$ be the Fredholm module associated to $M$ by Connes [5]. Let $P_{n}$ be the differential operator given by the relation

$$
\int_{M} B_{n}(f, h) d x=\int_{M} f P_{n}(h) d x
$$

for all $f, h \in C^{\infty}(M)$. Then,

i) $P_{n}$ is formally selfadjoint;

ii) $P_{n}$ is conformally invariant in the sense $\widehat{P_{n}}(h)=e^{-n \eta} P_{n}(h)$, if $\widehat{g}=e^{2 \eta} g$;

iii) $P_{n}$ is expressible universally as polynomial in the components of $\nabla$ (the covariant derivative) and $R$ (the curvature tensor) with coefficients rational in $n$.

iv) $P_{n}(h)=c_{n} \Delta^{n / 2}(h)+$ "lower order terms", with $c_{n}$ a universal constant; 
v) $P_{n}$ has the form $\delta S_{n} d$ where $S_{n}$ is an operator on 1-forms given as a constant multiple of $\Delta^{n / 2-1}+$ "lower order terms" or $(d \delta)^{n / 2-1}+$ "lower order terms";

vi) $P_{n}$ and $B_{n}$ are related by:

$$
P_{n}(f h)-f P_{n}(h)-h P_{n}(f)=-2 B_{n}(f, h) .
$$

Because the critical GJMS operator and the operator $P_{n}$ coincide in the flat case and share the same conformal behavior we have

Proposition 1. In the even dimensional case, inside the conformally flat class of metrics, the critical GJMS operator and the operator $P_{n}$ coincide up to a constant multiple.

\subsection{Yong Wang's work}

Based on the work of [20], in [21] and [22] Y. Wang proposes to extend to the case of manifolds with boundary, the work of Connes in Section 2 of [5]. He is the first to suggest the replacement of the usual noncommutative residue by the noncommutative residue of Fedosov-Golse-LeichtnamSchrohe [8], acting on Boutet de Monvel's algebra [3].

In Section 3 of [21], Y. Wang considers a compact $n$-dimensional manifold $X$ with boundary $Y$ and its double manifold $\widetilde{X}=X \cup_{Y} X$. For a vector bundle $E$ over $\widetilde{X}$ and a pseudodifferential operator $S$ with the transmission property and of order 0 acting on sections of $E$, the operator $\widetilde{P}$ is defined as the composition

$$
\widetilde{P}:=\left(\begin{array}{cc}
\pi^{+} f_{0} & 0 \\
0 & 0
\end{array}\right)\left[\left(\begin{array}{cc}
\pi^{+} S & 0 \\
0 & 0
\end{array}\right),\left(\begin{array}{cc}
\pi^{+} f_{1} & 0 \\
0 & 0
\end{array}\right)\right]\left[\left(\begin{array}{cc}
\pi^{+} S & 0 \\
0 & 0
\end{array}\right),\left(\begin{array}{cc}
\pi^{+} f_{2} & 0 \\
0 & 0
\end{array}\right)\right] .
$$

It is then observed that $\widetilde{P}=\pi^{+}\left(f_{0}\left[S, f_{1}\right]\left[S, f_{2}\right]\right)+G$ for some singular Green operator $G$ with singular Green symbol $b$. See [21] for the corresponding definitions. Based on this decomposition of $\widetilde{P}$ and the definition of Fedosov et al. of $\overline{\operatorname{Res}}$, Wang defines $\Omega_{n, S}$ and $\Omega_{n-1, S}$ via

$$
\Omega_{n, S}\left(f_{1}, f_{2}\right)=\overline{\operatorname{res}}\left(\left.\left(\left[S, \bar{f}_{1}\right]\left[S, \bar{f}_{2}\right]\right)\right|_{X}\right) \quad \text { and }\left.\quad f_{0}\right|_{Y} \Omega_{n-1, S}\left(f_{1}, f_{2}\right)=2 \pi \operatorname{res}_{x^{\prime}} \operatorname{trace}(b),
$$

with $\bar{f}_{i}$ a smooth extension of $f_{i}$ to $\widetilde{X}$. Also $\overline{\text { res }}$ is the density corresponding to $\overline{\operatorname{Res}}$ (similar to res for Res) and res $x^{\prime}$ the noncommutative residue density for the manifold $Y$.

It is possible to verify that $\widetilde{P}$ satisfies the transmission property. In this way, forgetting about any conformal invariance property, in [21] Wang found a generalization of the relation

$$
\operatorname{Res}\left(f_{0}\left[S, f_{1}\right]\left[S, f_{2}\right]\right)=\int_{M} f_{0} \Omega_{n, S}\left(f_{1}, f_{2}\right)=\int_{M} f_{0} B_{n, S}\left(f_{1}, f_{2}\right) d x, \quad \forall f_{i} \in C^{\infty}(X \cup Y)
$$

in Theorem 1 of [18].

The main idea of Y. Wang relies on the use of the double manifold. Topologically, the double manifold of a given compact oriented manifold with boundary makes perfect sense. At the level of smooth manifold with a given Riemannian structure more work is needed to make sense of a double manifold.

In Section 4 of [21] the dimension is taken as even and the metric on $X$ has a product structure near the boundary: $g^{X}=g^{\partial X}+d x^{n}$. On $\widetilde{X}$ the metric $\widetilde{g}$ is taken as $\widetilde{g}=g$ on both copies of $X$. Then $\Omega_{n}$ is defined as $\left.\Omega_{n, F}\left(\overline{f_{1}}, \overline{f_{2}}\right)\right|_{X}$ where $(\mathcal{H}, F)$ is the Fredholm module associated to $(\widetilde{X}, \widetilde{g})$, and $\overline{f_{i}}$ is an extension of $f_{i}$ to $\widetilde{M}$.

In [22] the even-dimensional Riemannian metric in consideration has the particular form $g^{X}=1 /\left(h\left(x_{n}\right)\right) g^{\partial X}+d x_{n}^{2}$ on a collar neighborhood $U$ of $\partial X$. Here $h$ is the restriction to $[0,1)$ of a smooth function $\widetilde{h}$ on $(-\varepsilon, 1)$ for some $\varepsilon>0$ such that $h(0)=1$ and $h\left(x_{n}\right)>0$. A metric $\hat{g}$ 
is associated to the double manifold $\widetilde{X}$ in the following way. Given $U$ and $\widetilde{h}$ as before, there is a metric $\hat{g}$ on $\widetilde{X}$ with the form $\hat{g}^{\widetilde{X}}=1 /\left(\widetilde{h}\left(x_{n}\right)\right) g^{\partial X}+d x_{n}^{2}$ on $U \cup_{\partial X} \partial X \times(-\varepsilon, 0]$ and such that $\left.\hat{g}\right|_{X}=g$.

Next, with $\left(\mathcal{H}, F_{\hat{g}}\right)$ the Fredholm module associated to $(\widetilde{X}, \hat{g}), \Omega_{n}$ and $\Omega_{n-1}$ are defined via the relation

$$
\overline{\operatorname{Res}}\left(\pi^{+} f_{0}\left[\pi^{+} F_{\hat{g}}, f_{1}\right]\left[\pi^{+} F_{\hat{g}}, f_{2}\right]\right)=\int_{X} f_{0} \Omega_{n}\left(f_{1}, f_{2}\right)(\hat{g})+\left.\int_{\partial M} f_{0}\right|_{\partial X} \Omega_{n-1}(\hat{g}) .
$$

The described settings used in [21] and [22] have the following limitations: if we conformally rescale the metric in $X$ then, the new metric $e^{2 \eta} g$ is not anymore of the specific requested form near the boundary. How to define then the objects in question in terms of this new metric? That is to say, what is the definition of $\Omega_{n}\left(e^{2 \eta} g\right)$ and how to compare it with $\Omega_{n}(g)$ ? Based on the idea of replacing Res with $\overline{\operatorname{Res}}$, and inspired by the work of Wang, we propose the approach in this work to the problem of extending the results in Section 2 of [5] to manifolds with boundary.

\subsection{Contents}

We first review the construction of the even Fredholm module $(\mathcal{H}, F, \gamma)$ over the commutative algebra $\mathcal{A}=C^{\infty}(M)$ (trivially an involutive algebra over $\mathbb{C}$ ) of smooth functions over a compact oriented manifold $M$ without boundary. We give special attention to its conformal properties. Then we review the statement of Connes about recovering the conformal structure from this Fredholm module and a recent characterization on the subject by Bär. Next, we move to the setting of manifolds with boundary. Aiming to extend previous work, we briefly review the noncommutative residue for manifolds with boundary and Boutet de Monvel calculus according to our needs. Last, we present a couple of results that extend to the even dimensional case Theorems 1 and 2 in [18] to the following setting of manifolds with boundary: $M$ is a compact manifold with boundary $\partial M$ such that $M$ is embedded in a compact oriented manifold $\widetilde{M}$ without boundary. Further we assume Riemannian structures $(\widetilde{M}, \widetilde{g})$ and $(M, g)$ such that $g$ coincides with $\widetilde{g}$ restricted to $M$. The results are Theorems 4 and 5 respectively.

\subsection{Other possibilities}

For a Riemann surface $M$, a map $f=\left(f^{i}\right)$ from $M$ to $\mathbb{R}^{2}$ and metric $g_{i j}(x)$ on $M$, the 2-dimensional Polyakov action [15] is given by

$$
I(f)=\frac{1}{2 \pi} \int_{M} g_{i j} d f^{i} \wedge \star d f^{j} .
$$

By considering instead of $d f$ its quantized version $[F, f]$, Connes [5] quantized the Polyakov action as a Dixmier trace:

$$
\frac{1}{2 \pi} \int_{M} g_{i j} d f^{i} \wedge \star d f^{j}=-\frac{1}{2} \operatorname{Tr}_{\omega}\left(g_{i j}\left[F, f^{i}\right]\left[F, f^{j}\right]\right) .
$$

Connes' trace theorem [4] states that the Dixmier trace and the noncommutative residue of an elliptic pseudodifferential operator of order $-n$ on an $n$-dimensional manifold $M$ are proportional by a factor of $n(2 \pi)^{n}$. In the 2-dimensional case the factor is $8 \pi^{2}$ and so, the quantized Polyakov action can be written as

$$
-16 \pi^{2} I=\operatorname{Res}\left(g_{i j}\left[F, f^{i}\right]\left[F, f^{j}\right]\right) .
$$

This quantized Polyakov action makes sense in the general even dimensional case. 
Although this generalization of the Polyakov action motivates the particular form of the functional $\operatorname{Res}\left(f_{0}\left[F, f_{1}\right]\left[F, f_{2}\right]\right)$, the same Fredholm module yields other functionals in dimensions greater than 4 . For instance, for dimension 6 one could also consider

$$
\operatorname{Res}\left(f_{0}\left[F, f_{1}\right]\left[F, f_{2}\right]\left[F, f_{3}\right]\right)=\int_{M} f_{0} T\left(f_{1}, f_{2}, f_{3}\right) d^{6} x
$$

which is a Hochschild 3-cocycle and the trilinear expression $T\left(f_{1}, f_{2}, f_{3}\right)$ is conformally invariant. In greater $2 l$ dimensions,

$$
\operatorname{Res}\left(f_{0}\left[F, f_{1}\right] \cdots\left[F, f_{l}\right]\right)=\int_{M} f_{0} C\left(f_{1}, \ldots, f_{l}\right) d^{2 l} x,
$$

invites to study the role of the conformal invariant $C\left(f_{1}, \ldots, f_{l}\right)$.

\subsection{Further directions}

The possibility of obtaining from the expression

$$
\begin{array}{r}
\int_{M} B_{n}\left(f_{1}, f_{2}\right) d x+2 \pi \int_{\partial M} \partial B_{n}\left(\left.f_{1}\right|_{\partial M},\left.f_{2}\right|_{\partial M}\right) d x^{\prime} \\
=\int_{M} f_{1} P_{n} f_{2} d x+\left.\left.\int_{\partial M} f_{1}\right|_{\partial M} P_{n-1}^{\prime} f_{2}\right|_{\partial M} d x^{\prime}
\end{array}
$$

conformally covariant differential operators $P_{n}$ and $P_{n-1}^{\prime}$ acting on $M$ and $\partial M$ respectively (in a way similar to the boundaryless case), is the motivating force behind this project. We hope to report on that in the near future.

One more possibility is to study a Riemannian manifold with a particular metric structure near the boundary, in such a way that it makes sense to consider its double manifold and at the same time, study conformal variations of the metric. One can ask what sort of specific objects are to be found using the ideas presented here in such a particular situation. A seemingly promising case is that of manifolds with totally geodesic boundaries, for which the double manifold is natural to be considered.

\section{The Fredholm module for a conformal manifold}

Following Definition IV.4.1 [6], an even Fredholm module $(\mathcal{H}, F, \gamma)$ is given by

- An involutive algebra $\mathcal{A}$ (over $\mathbb{C}$ ) together with a Hilbert space $\mathcal{H}$ and an involutive representation $\pi$ of $\mathcal{A}$ in $\mathcal{H}$.

- An operator $F$ on $\mathcal{H}$ such that $F=F^{*}, F^{2}=1$, and $[F, \pi(a)]$ is a compact operator $\forall a \in \mathcal{A}$.

- A $\mathbb{Z} / 2$ grading $\gamma, \gamma=\gamma^{*}, \gamma^{2}=1$ of $\mathcal{H}$ such that $\gamma \pi(a)=\pi(a) \gamma, \forall a \in \mathcal{A}$, and $\gamma F=-F \gamma$.

In the case of a manifold without boundary, the very first ingredient in $(\mathcal{H}, F, \gamma)$ is the involutive algebra $\mathcal{A}=C^{\infty}(M)$ where we allow complex values. The fact that, for an oriented Riemannian manifold $M$ (with or without boundary) and of even dimension $n$, the restriction of the Hodge star operator to middle-dimension forms is conformally invariant, is central to what follows. We consider the vector bundle $\Omega_{\mathbb{C}}^{n / 2}(M)$ of complex middle-dimension forms. We drop the subscript $\mathbb{C}$ from now on. In this way, for an $n$-dimensional oriented compact manifold, $n$ even, the space $\Omega^{n / 2}(M)$ of (complex) middle dimension forms has a (complexified) inner product

$$
\left\langle\omega_{1}, \omega_{2}\right\rangle=\int_{M} \overline{\omega_{1}} \wedge \star \omega_{2}
$$


This inner product is unchanged under a conformal change of the metric and so, its Hilbert space completion $\mathcal{H}_{0}=L^{2}\left(M, \Omega^{n / 2}(M)\right)$ depends only on the conformal class of the metric. $\mathcal{H}_{0}$ is by construction a $C^{\infty}(M)$-module with $(f \omega)(p)=f(p) \omega(p)$ for all $f \in C^{\infty}(M), \omega \in \mathcal{H}_{0}$, and $p \in M$.

If $M$ is a compact manifold without boundary then, the harmonic forms (those in the kernel of $\Delta$ ) are precisely those in $\operatorname{Ker} d \cap \operatorname{Ker} \delta$. If $M$ is even dimensional with dimension $n$, the Hodge decomposition for middle-dimension forms looks like

$$
\Omega^{n / 2}(M)=\Delta\left(\Omega^{n / 2}(M)\right) \oplus H^{n / 2}=d\left(\Omega^{n / 2-1}(M)\right) \oplus \delta\left(\Omega^{n / 2+1}(M)\right) \oplus H^{n / 2} .
$$

Here $H^{n / 2}=\operatorname{Ker}_{n / 2} \Delta$. Thus $\mathcal{H}_{0}$ is the direct sum of $H^{n / 2}$ and the images of $d$ and $\delta$.

The Hilbert space $\mathcal{H}$ is $\mathcal{H}=\mathcal{H}_{0} \oplus H^{n / 2}$, the direct sum of $\mathcal{H}_{0}$ with an extra copy of the finite dimensional Hilbert space of harmonic middle-dimension forms on $M$.

For each $f \in \mathcal{A}=C^{\infty}(M)$ we consider the multiplication operator on $\mathcal{H}_{0}, f: \omega \mapsto f \omega$. These multiplication operators on $\mathcal{H}_{0}$ do not preserve the subspace of harmonic forms. Thus the extra copy of $H^{n / 2}$ in $\mathcal{H}$ is to preserve the notion of $\mathbb{Z}_{2}$-graded Hilbert space.

The Hilbert space representation of $C^{\infty}(M)$ in $\mathcal{H}$ is given by $f \mapsto \pi(f)$ with $\pi(f)(\omega+h):=f \omega$, for all $\omega \in \mathcal{H}_{0}$ and $h \in H^{n / 2}$. Evidently, $\pi(f)$ is a bounded operator on $\mathcal{H}$. It is not difficult to verify that $\pi$ is an involutive representation of $C^{\infty}(M)$ in $\mathcal{H}$. To simplify the notation we write $f$ instead of $\pi(f)$.

Next we look at the $\mathbb{Z} / 2$ grading. Because the Hodge star operator $\star$ acting on middledimension forms satisfies $\star^{2}=(-1)^{n / 2}$, the operator

$$
\gamma_{0}:=(-1)^{\frac{(n / 2)(n / 2-1)}{2}} i^{n / 2} \star
$$

is of square one giving a $\mathbb{Z}_{2}$-grading on $\mathcal{H}_{0}$. Since $\star^{*}=(-1)^{n / 2} \star$ when acting on middle forms we have

$$
\gamma_{0}^{*}:=(-1)^{\frac{(n / 2)(n / 2-1)}{2}}(-i)^{n / 2} \star^{*}=(-1)^{\frac{(n / 2)(n / 2+3)}{2}} i^{n / 2} \star=\gamma_{0} .
$$

It follows from the definition of $\gamma_{0}$ that

Lemma 1. The operator $\gamma_{0}$ exchanges the subspaces $d\left(\Omega^{n / 2-1}\right)$ and $\delta\left(\Omega^{n / 2+1}\right)$ and their closures. As a consequence and because of $(1), \gamma_{0}\left(H^{n / 2}\right)=H^{n / 2}$.

We define $\gamma: \mathcal{H}_{0} \oplus H^{n / 2} \rightarrow \mathcal{H}_{0} \oplus H^{n / 2}$ by $\gamma(\omega+h):=\gamma_{0}(\omega)-\gamma_{0} h$. In this way, the extra copy of $H^{n / 2}$ is endowed with the opposite $\mathbb{Z}_{2}$-grading $-\gamma: H^{n / 2 \pm}=\left\{h \in H^{n / 2}: \gamma h=\mp h\right\}$ and so $\mathcal{H}$ has the $\mathbb{Z}_{2}$-grading given by $\mathcal{H}^{+}=\mathcal{H}_{0}^{+} \oplus H^{n / 2^{+}}$and $\mathcal{H}^{-}=\mathcal{H}_{0}^{-} \oplus H^{n / 2^{-}}$.

It is straightforward to verify that the operator $\gamma$ defined on $\mathcal{H}$ satisfies $\gamma=\gamma^{*}, \gamma^{2}=1$, and $\gamma f=f \gamma$ for all $f \in C^{\infty}(M)$.

The first step to define the operator $F$ is the following observation

Lemma 2. For $\omega=d \beta+\delta \beta^{\prime} \in d\left(\Omega^{n / 2-1}(M)\right) \oplus \delta\left(\Omega^{n / 2+1}(M)\right)$, the operator $F_{0}: \mathcal{H}_{0} \rightarrow \mathcal{H}_{0}$ defined by $F_{0}\left(d \beta+\delta \beta^{\prime}\right):=d \beta-\delta \beta^{\prime}$ and extended as zero over $H^{n / 2}$ is a partial isometry such that $F_{0}^{2}=1$ on $\mathcal{H}_{0} \ominus H^{n / 2}$, and $F_{0}$ is its own formal adjoint operator. Furthermore, $1-F_{0}^{2}$ is the orthogonal projection on the finite-dimensional Hilbert space of middle-dimension harmonic forms.

The operator $F$ is defined on $\mathcal{H}=d\left(\Omega^{n / 2-1}(M)\right) \oplus \delta\left(\Omega^{n / 2+1}(M)\right) \oplus H^{n / 2} \oplus H^{n / 2}$ by

$$
F=\left(\begin{array}{ccc}
F_{0} & 0 & 0 \\
0 & 0 & 1 \\
0 & 1 & 0
\end{array}\right) .
$$

From the previous lemma $F^{*}=F$ and $F^{2}=1$. 
For an even dimensional oriented compact manifold without boundary, both the Hilbert space $\mathcal{H}$ and the operator $F$ are conformally invariant, thanks to the fact: for a k-form $\rho$,

$$
\widehat{\delta} \rho=e^{-(n-2(k-1)) \eta} \delta e^{(n-2 k) \eta} \rho .
$$

It is not difficult to verify that $\gamma F=-F \gamma$. Last, since each $[F, f]$ is a pseudodifferential operator of order -1 for all $f \in \mathcal{A}=C^{\infty}(M)$, the operator $[F, f]$ is a compact operator on $\mathcal{H}$ via Rellich's theorem [9, p. 306].

\subsection{Recovering the conformal structure}

Theorem IV.4.2.c of [6] states that the Fredholm module $(\mathcal{H}, F)$ uniquely determines the conformal structure of $M$. For that, Connes uses his trace theorem and the noncommutative residue to recover the $L^{n}$-norm for exterior 1 -forms over the manifold.

The first step is to consider instead of $d f$ its quantized version $[F, f]$. Since $F$ is a pseudodifferential operator of order $0,[F, f]$ is a pseudodifferential operator of order -1 for all $f \in C^{\infty}\left(M^{n}\right)$, acting on the same vector bundle $\Omega^{n / 2} M$ as $F$. The leading symbol of $F$ is given by

$$
\sigma_{0}(F)(x, \xi)=|\xi|^{-2}\left(\varepsilon \frac{n}{2}-1(\xi) \iota \frac{n}{2}(\xi)-\iota_{\frac{n}{2}+1}(\xi) \varepsilon_{\frac{n}{2}}(\xi)\right)
$$

for all $(x, \xi) \in T^{*} M, \xi \neq 0$. Here $\varepsilon_{k}(\xi)$ and $\iota_{k}(\xi)$ represent the exterior and interior multiplication by the 1 -form $\xi$ on $k$-forms. Note how $\sigma_{0}(F)$ does not depend on $x \in M$. The principal symbol of $[F, f]$ is

$$
\sigma_{-1}([F, f])(x, \xi)=-i \sum_{k=1}^{n} \partial_{x^{k}} f \partial_{\xi_{k}}\left(\sigma_{0}(F)\right)
$$

which by the expression for $\sigma_{0}(F)(x, \xi)$ depends only on the value on $x$ of the 1 -form $d f=$ $\sum \partial_{x^{k}} f d x^{k}$. The details of these statements can be seen for example in [20]. Next for $f_{i} \in$ $C^{\infty}(M)$ the operator $\left(f_{1}\left[F, f_{2}\right]\right)^{n}$ is a pseudodifferential operator of order $-n$.

What Theorem IV.4.2.c [6] shows is that $\operatorname{Res}\left(f_{1}\left[F, f_{2}\right]\right)^{n}$ and $\int_{M}\left\|f_{1} d f_{2}\right\|^{n} d x$, the $L^{n}$-norm for 1-forms, are proportional.

In the setting of spin Riemannian manifolds, for the algebra $C^{\infty}(M)$ of smooth complex valued functions, the Hilbert space is chosen to be $\mathcal{H}=L^{2}(M, \Sigma M)$, the square integrable complex spinor fields, and for $F$ one considers the sign of the Dirac operator $D$. Recently, Bär [2] showed the following result.

Theorem 2. Let $M$ be a compact spin Riemannian manifold. Let $g$ and $g^{\prime}$ be Riemannian metrics on $M$ and let $(\mathcal{H}, \operatorname{sign}(D))$ and $\left(\mathcal{H}^{\prime}, \operatorname{sign}\left(D^{\prime}\right)\right)$ be the corresponding Fredholm modules of the algebra $C^{\infty}(M)$. Then $g$ and $g^{\prime}$ are conformally equivalent if and only if $(\mathcal{H}, \operatorname{sign}(D))$ and $\left(\mathcal{H}^{\prime}, \operatorname{sign}\left(D^{\prime}\right)\right)$ are weakly unitarily equivalent. That is to say, there is a unitary isomorphism $U: \mathcal{H} \rightarrow \mathcal{H}^{\prime}$ such that $D^{\prime}-U D U^{-1}$ is a compact operator and for all $f \in C^{\infty}(M)$ and all $h \in \mathcal{H}$ one has $U(f h)=f U(h)$.

The idea is based on the commutativity of $U$ with the action of $C^{\infty}(M)$ which implies that $U$ is induced by a (a.e. invertible) section $\Psi$ of $L^{\infty}\left(M, \operatorname{Hom}\left(\Sigma M, \Sigma^{\prime} M\right)\right)$. The principal symbol of a Dirac operator is given by Clifford multiplication with respect to the metric $g$, $\sigma_{D}(\xi)=i c_{g}(\xi), \quad$ for all $\xi \in T^{*} M$. Because of the relation $c_{g}(\xi) c_{g}(\eta)+c_{g}(\eta) c_{g}(\xi)=-2 g(\xi, \eta)$, for all $\xi, \eta \in T^{*} M$, the principal symbol of $\operatorname{sign}(D)$ is

$$
\sigma_{\operatorname{sign}(D)}(\xi)=\frac{i c_{g}(\xi)}{\|\xi\|_{g}}, \quad \forall \xi \in T^{*} M \backslash\{0\} .
$$


Since $D^{\prime}$ and $U D U^{-1}$ differ by a compact operator, they have the same sign and thus

$$
\frac{c_{g^{\prime}}(\xi)}{\|\xi\|_{g^{\prime}}}=\Psi(x) \frac{c_{g}(\xi)}{\|\xi\|_{g}} \Psi^{-1}(x)
$$

for all nonzero $\xi \in T^{*} M$. Last

$$
\begin{aligned}
\frac{-2 g^{\prime}(\xi, \eta)}{\|\xi\|_{g^{\prime}}\|\eta\|_{g^{\prime}}} & =\frac{c_{g^{\prime}}(\xi) c_{g^{\prime}}(\eta)+c_{g^{\prime}}(\eta) c_{g^{\prime}}(\xi)}{\|\xi\|\left\|_{g^{\prime}}\right\| \eta \|_{g^{\prime}}} \\
& =\Psi(x)\left(\frac{c_{g}(\xi) c_{g}(\eta)+c_{g}(\eta) c_{g}(\xi)}{\|\xi\|_{g}\|\eta\|_{g}}\right) \Psi^{-1}(x)=\frac{-2 g(\xi, \eta)}{\|\xi\|_{g}\|\eta\|_{g}}
\end{aligned}
$$

since the term in the middle is a scalar.

It is important to recall here the result of Connes (see for example [6, p. 544]) that says that one recovers the metric distance between points in a connected manifold $(M, g)$ from the relation

$$
d(x, y)=\sup \left\{f(x)-f(y): f \in C^{\infty}(M) \text { with }\|[D, f]\| \leq 1\right\} .
$$

Note how with the stronger requirement $D^{\prime}=U D U^{-1}$ (unitarily equivalent) then $\|[D, f]\|=$ $\left\|\left[D^{\prime}, f\right]\right\|$ and thus $d=d^{\prime}$.

If the conformal geometry of $(M,[g])$ is encoded in the Fredholm module $(\mathcal{H}, F, \gamma)$ over the algebra $C^{\infty}(M)$, then how can one extract the conformal geometry from this Fredholm module?

One possibility is to use it to find conformal invariants associated to a given conformal manifold, for example, as in the introduction.

\section{The noncommutative residue for manifolds with boundary}

Remark 1. Wodzicki: (see e.g. [16]) There is no non-zero trace on the algebra of classical pseudodifferential operators mod the ideal of smoothing operators $\Psi^{\infty}(M) / \Psi^{-\infty}(M)$, whenever $M$ is noncompact or has a boundary.

The noncommutative residue of Fedosov-Golse-Leichtnam-Schrohe [8] for manifolds with boundary is the unique (up to a constant multiple) continuous trace for the operators in Boutet de Monvel's algebra. Roughly speaking, this noncommutative residue acts on operators $A$ that are described by pairs of symbols $\left\{a_{i}, a_{b}\right\}$ called interior and boundary symbol respectively. In case the manifold has empty boundary this noncommutative residue coincides with the usual noncommutative residue of Wodzicki, Guillemin, Adler, and Manin.

The setting for the noncommutative residue is given by a compact manifold $M$ with boundary $\partial M$ such that $M$ is embedded in a compact manifold $\widetilde{M}$ without boundary, both $M$ and $\widetilde{M}$ of dimension $n>1$. For $M$ we consider in a boundary chart local coordinates given by $\left(x^{\prime}, x^{n}\right)$ with $x^{\prime}=\left(x^{1}, \ldots, x^{n-1}\right)$ coordinates for $\partial M$ and $x^{n}$ the geodesic distance to $\partial M$. It is important to mention that the geodesic coordinate chosen for $x^{n}$ is only a technical tool since the noncommutative residue is independent of the metric and of local representations.

\subsection{Boutet de Monvel's sub-algebra of diagonal symbols}

In $[3,8,11]$, and [16] one can find detailed introductions to Boutet de Monvel's calculus. The operators in Boutet de Monvel's algebra we are interested in are diagonal matrices of operators (endomorphisms) $A$ acting on sections of vector bundles $E$ over $M$ and $E^{\prime}$ over $\partial M$ :

$$
A=\left(\begin{array}{cc}
r^{M} P e^{M}+G & 0 \\
0 & S
\end{array}\right): \underset{C^{\infty}\left(\partial M, E^{\prime}\right)}{C^{\infty}(M, E)} \rightarrow \begin{gathered}
C^{\infty}(M, E) \\
C^{\infty}\left(\partial M, E^{\prime}\right)
\end{gathered}
$$


They are better described by a pair of symbols $\left(a_{i}, a_{b}\right)$ where $a_{i}$ is called the interior symbol and $a_{b}$ is called the boundary symbol. According to our needs, the characterization of such an operator (or its symbol) of order $m$ is as follows.

P. The operator $P$ is a classical pseudodifferential operator of order $m$ on $\widetilde{M}$. Furthermore, $P$ has the so called transmission property. This guarantees that the composition of different elements remains inside the algebra. Analytically, in local coordinates near $\partial M$ the transmission property is given by

$$
\partial_{x^{n}}^{k} \partial_{\xi^{\prime}}^{\alpha} p_{j}\left(x^{\prime}, 0,0,+1\right)=(-1)^{j-|\alpha|} \partial_{x^{n}}^{k} \partial_{\xi^{\prime}}^{\alpha} p_{j}\left(x^{\prime}, 0,0,-1\right), \quad \forall j, k, \alpha .
$$

Here $p_{j}$ is the homogeneous component of order $j$ in the symbol expansion of the symbol $p$ of $P$. Last, $e^{M}$ is the extension by zero of functions (or sections) on $M$ to functions (or sections) on $\widetilde{M}$ and $r^{M}$ is the restriction from $\widetilde{M}$ to $M$. The interior symbol $a_{i}$ of $A$ is precisely $p$.

With $\mathcal{F}$ we denote the Fourier transform. Also

$$
\begin{aligned}
& H^{+}=\left\{\mathcal{F}\left(\chi_{] 0, \infty} u\right): u \text { is a rapidly decreasing function on } \mathbb{R}\right\}, \\
& H_{0}^{-}=\left\{\mathcal{F}\left(\left(1-\chi_{] 0, \infty}\right) u\right): u \text { is a rapidly decreasing function on } \mathbb{R}\right\}, \\
& H^{-}=H_{0}^{-} \oplus\{\text { all polynomials }\} .
\end{aligned}
$$

The (diagonal) boundary symbol $a_{b}$ is given by a pair of symbols $b, s$ of operators $G, S$ parametrized by $T^{*} \partial M \backslash\{0\}$ and the restriction of $p$ to the boundary.

G. The operator $G$ is given by a singular green operator-symbol $b\left(x^{\prime}, \xi^{\prime}, D_{n}\right)$ in the following way. For every $l$ and fixed $x^{\prime}, \xi^{\prime}$,

$$
b_{l}\left(x^{\prime}, \xi^{\prime}, \xi_{n}, \eta_{n}\right) \in H^{+} \hat{\otimes}_{\pi} H^{-} .
$$

With $\hat{\otimes}_{\pi}$ we denote Grothendieck's completion of the algebraic tensor product. The operator $b\left(x^{\prime}, \xi^{\prime}, D_{n}\right): H^{+} \rightarrow H^{+}$is given by

$$
\left[b\left(x^{\prime}, \xi^{\prime}, D_{n}\right) h\right]\left(\xi_{n}\right)=\Pi_{\eta_{n}}^{\prime}\left(b\left(x^{\prime}, \xi^{\prime}, \xi_{n}, \eta_{n}\right) h\left(\eta_{n}\right)\right)=\lim _{\eta_{n} \rightarrow 0^{+}} \mathcal{F}^{-1}\left(b\left(x^{\prime}, \xi^{\prime}, \xi_{n}, \cdot\right) h(\cdot)\right)\left(\eta_{n}\right) .
$$

The operator $G$ described by this operator-symbol $b\left(x^{\prime}, \xi^{\prime}, D_{n}\right)$ between functions on $[0, \infty[$ that are rapidly decreasing at $\infty$, defines a trace class operator on $L^{2}\left(R_{+}\right)$. The trace is given by

$$
\operatorname{trace}(G)\left(x^{\prime}, \xi^{\prime}\right)=\frac{1}{2 \pi} \int b\left(x^{\prime}, \xi^{\prime}, \xi_{n}, \xi_{n}\right) d \xi_{n} .
$$

Note that this is actually a symbol itself.

S. The operator $S$ is a classical pseudodifferential operator of order $m$ along the boundary. It has values in $\mathcal{L}\left(\mathbb{C}^{k}\right)$ and each component $s_{j}$ of its symbol expansion $s$ acts by multiplication on $\mathbb{C}^{k}$.

The (diagonal) boundary symbol $a_{b}$ is then

$$
a_{b}\left(x^{\prime}, \xi^{\prime}, \xi_{n}, \eta_{n}\right)=\left(\begin{array}{cc}
p\left(x^{\prime}, 0, \xi^{\prime}, \xi_{n}\right)+b\left(x^{\prime}, \xi^{\prime}, \xi_{n}, \eta_{n}\right) & 0 \\
0 & s\left(x^{\prime}, \xi^{\prime}\right)
\end{array}\right)
$$


with

$$
b\left(x^{\prime}, \xi^{\prime}, \xi_{n}, \eta_{n}\right) \sim \sum_{l=-\infty}^{m} b_{l}\left(x^{\prime}, \xi^{\prime}, \xi_{n}, \eta_{n}\right) \quad \text { and } \quad s\left(x^{\prime}, \xi^{\prime}\right) \sim \sum_{l=-\infty}^{m} s_{l}\left(x^{\prime}, \xi^{\prime}\right)
$$

where for $\lambda>0$

$$
b_{l}\left(x^{\prime}, \lambda \xi^{\prime}, \lambda \xi_{n}, \lambda \eta_{n}\right)=\lambda^{l} b_{l}\left(x^{\prime}, \xi^{\prime}, \xi_{n}, \eta_{n}\right), \quad s_{l}\left(x^{\prime}, \lambda \xi^{\prime}\right)=\lambda^{l} s_{l}\left(x^{\prime}, \xi^{\prime}\right) .
$$

By $\mathcal{B}_{D}^{m}(M)$ we denote the collection of all operators of order $m$ with diagonal boundary symbol and by $\mathcal{B}_{D}^{\infty}(M)$ the union of all the $\mathcal{B}_{D}^{m}(M)$. The intersection over all orders $m$ of $\mathcal{B}_{D}^{m}(M)$ is denoted $\mathcal{B}_{D}^{-\infty}(M)$. Last $\mathcal{B}_{D}=\mathcal{B}_{D}^{\infty}(M) / \mathcal{B}_{D}^{-\infty}(M)$.

Given two operators $A_{1}$ and $A_{2}$ in $\mathcal{B}_{D}$ with symbols $\left(a_{i 1}, a_{b 1}\right)$ and $\left(a_{i 2}, a_{b 2}\right)$, with entries in the boundary symbols $b_{j}, s_{j}$, for $j=1,2$, the composition is again an operator in $\mathcal{B}_{D}$ with symbol $\left(a_{i}, a_{b}\right)$ where $a_{i}$ is the usual composition of symbols $a_{i}=a_{i 1} \circ a_{i 2}$. It also satisfies the transmission property.

The resulting boundary symbol is of the form

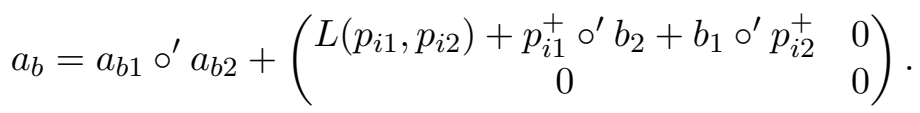

The symbol o' denotes the usual composition of pseudodifferential symbols on the variables $\left(x^{\prime}, \xi^{\prime}\right)$. The terms in the second summand represent the portion on the boundary symbol coming from the interior symbols. Here, we have hidden in $a_{b 1} \circ^{\prime} a_{b 2}$ the part corresponding to the restriction to the boundary of the interior symbol.

The so called "left-over term" $L\left(p_{i 1}, p_{i 2}\right)$, reflects the particular way the pseudodifferential operators $P_{M}=r^{M} P e^{M}$ act on the manifold with boundary $M$. If $P_{1}$ and $P_{2}$ are two pseudodifferential operators on $\widetilde{M}$, the difference $\left(P_{1} P_{2}\right)_{M}-\left(P_{1}\right)_{M}\left(P_{2}\right)_{M}$ is a singular Green operator with associated singular Green operator-symbol $L\left(p_{1}, p_{2}\right)$. Since this left-over term need not be zero, we can not reduce the diagonal sub-algebra by requesting $G=0$ in all the operators.

As an example, and because they will be needed later on, let us look at $L(f, q)$ and $L(p, f)$ where $p$ and $q$ are the symbols of pseudodifferential operators $P$ and $Q$ on $\widetilde{M}$, and $f \in C^{\infty}(\widetilde{M})$, i.e. $f$ represents the pseudodifferential operator on $\widetilde{M}$ given multiplication by $f$. Among all the possible formulae for $L(p, q)$ available in the literature we decided to use the one provided in [11].

In Section 3 of [11] one can read an explicit expression for $L(p, q)$ in which the effects of $p$ and $q$ are neatly separated. This expression uses singular Green operators $G^{+}(p)$ and $G^{-}(q)$ natural for the calculus in use (see Theorems 3.2 and 3.4 [11]).

We content ourselves by quoting a particular situation. By Theorem $3.4[11], G^{-}(f)=0$ and by $(3.16)[11], L(p, f)=G^{+}(p) G^{-}(f)$, thus $L(p, f)=0$.

Now, for $L(f, q)$ we must look at Theorem 3.5 [11]. In general,

$$
L(p, q)=G^{+}(p) G^{-}(q)+\sum_{0 \leq m<\text { order of } Q} K_{m} \gamma_{m}
$$

where the $K_{m}$ are operators obtained from symbols of a particular type known as Poisson symbols. By (3.35) [11], $K_{m}=0$ when $p=f$ since it depends on higher derivatives on $\xi_{n}$. Since by Theorem $3.2[11], G^{+}(f)=0$ we conclude that $L(f, p)=0$ as well.

Lemma 3. For every $f \in C^{\infty}(\widetilde{M})$ and every pseudodifferential operator $P$ on $\widetilde{M}$ with symbol $p$, both left-over terms $L(f, p)$ and $L(p, f)$ vanish.

Last, the operator $p^{+}\left(x^{\prime}, \xi^{\prime}, D_{n}\right): H^{+} \rightarrow H^{+}$is induced from the action (of the interior symbol) in the normal direction for fixed $\left(x^{\prime}, \xi^{\prime}\right)$. The only case we will be interested in are those of the form $f^{+} o^{\prime} b_{2}$ where $f$ is a smooth function on $\widetilde{M}$. We will address them in (3). 


\subsection{The noncommutative residue}

On $\mathbb{R}^{n}$ with coordinates $\xi_{1}, \ldots, \xi_{n}$ we consider the $(n-1)$-form

$$
\sigma=\sum_{j=1}^{n}(-1)^{j+1} \xi_{j} d \xi_{1} \wedge \cdots \wedge \widehat{d \xi}_{j} \wedge \cdots \wedge d \xi_{n}
$$

where the hat indicates this factor is omitted. Restricted to the unit sphere $\mathbb{S}^{n-1}, \sigma$ gives the volume form on $\mathbb{S}^{n-1}$ and in general $d \sigma=n d \xi_{1} \wedge \cdots \wedge d \xi_{n}$. For a coordinate chart $U$, the form $d x_{1} \wedge \cdots \wedge d x_{n}$ defines an orientation on $U$ and induces the orientation $d \xi_{1} \wedge \cdots \wedge d \xi_{n}$ on $\mathbb{R}^{n}$.

For a closed compact manifold $M$ without boundary, the noncommutative residue is defined as the unique trace (up to constant multiples) on the algebra $\Psi^{\infty} / \Psi^{-\infty}$ of classical pseudodifferential operators mod the ideal of smoothing operators.

The following is the main result of [8]:

Theorem 3 (Fedosov-Golse-Leichtnam-Schrohe). Let $M$ be a manifold of dimension $n$ with smooth boundary $\partial M$, and let $M \cup \partial M$ be embedded in a connected manifold $\widetilde{M}$ of dimension $n$. Let

$$
A=\left(\begin{array}{cc}
r^{M} P e^{M}+G & K \\
T & S
\end{array}\right)
$$

be an element in $\mathcal{B}^{\infty}(M) / \mathcal{B}^{-\infty}(M)$, with $\mathcal{B}^{\infty}(M)$ the algebra of all operators in Boutet de Monvel's calculus (with integral order), $\mathcal{B}^{-\infty}(M)$ the ideal of smoothing operators, and let $p, b$, and $s$ denote the local symbols of $P, G$, and $S$ respectively. Then

$$
\begin{aligned}
\overline{\operatorname{Res}} A= & \int_{M} \int_{S^{n-1}} \operatorname{Tr}_{E} p_{-n}(x, \xi) \sigma(\xi) d x \\
& +2 \pi \int_{\partial M} \int_{S^{n-2}}\left\{\operatorname{Tr}_{E^{\prime}}\left(\operatorname{trace} b_{-n}\right)\left(x^{\prime}, \xi^{\prime}\right)+\operatorname{Tr}_{E^{\prime}} s_{1-n}\left(x^{\prime}, \xi^{\prime}\right)\right\} \sigma^{\prime}\left(\xi^{\prime}\right) d x^{\prime},
\end{aligned}
$$

with $\sigma^{\prime}$ the $n-2$ analog of $\sigma$, is the unique continuous trace (up to constant multiples) on the algebra $\mathcal{B}^{\infty}(M) / \mathcal{B}^{-\infty}(M)$.

This trace reduces to the noncommutative residue (of Adler, Manin, Guillemin, and Wodzicki) in the case $\partial M=\varnothing$, and it is independent of the Riemannian metric (eventually) chosen on $M$.

\section{On manifolds with boundary}

In this section we present an extension of Theorem 1 in [18] to the setting of manifolds with boundary. Let $M$ be a manifold with boundary $\partial M$. Assume that the compact manifold $M$ is embedded in a compact manifold $\widetilde{M}$ without boundary. Further we assume $\widetilde{M}$ to be oriented which determines an orientation on $M$ and thus on $\partial M$.

For $P$ a pseudodifferential operator acting on a vector bundle $E$ over $\widetilde{M}$ with symbol $p$ having the transmission property up to the boundary, $S$ a pseudodifferential operator acting on a vector bundle $E^{\prime}$ over $\partial M$ with symbol $s$, and for $f \in C^{\infty}(\widetilde{M})$ we let $A(P, S)$ and $A(f)$ be the elements in Boutet de Monvel's algebra of diagonal elements given by

$$
A(P, S)=\left(\begin{array}{cc}
r^{M} P e^{M}+0 & 0 \\
0 & S
\end{array}\right), \quad A(f)=\left(\begin{array}{cc}
r^{M} f e^{M}+0 & 0 \\
0 & \left.f\right|_{\partial M}
\end{array}\right) .
$$

We study $\operatorname{Res}\left(A\left(f_{0}\right)\left[A(P, S), A\left(f_{1}\right)\right]\left[A(P, S), A\left(f_{2}\right)\right]\right)$ for functions $f_{i} \in C^{\infty}(\widetilde{M})$. 
First of all, we must check that this product operator remains inside the calculus in use. It follows from Proposition 2.7 of [17], which states that if two operators satisfy the transmission property then their product satisfies the transmission property as well.

Since $L(f, p)=0=L(p, f)$, for all $f \in C^{\infty}(\widetilde{M})$ it follows that

$$
\begin{aligned}
& A\left(f_{0}\right)\left[A(P, S), A\left(f_{1}\right)\right]\left[A(P, S), A\left(f_{2}\right)\right]= \\
& \left(\begin{array}{cc}
r^{M} f_{0}\left[P, f_{1}\right]\left[P, f_{2}\right] e^{M}+f_{0}^{+} \circ^{\prime} L\left(\sigma\left(\left[P, f_{1}\right]\right), \sigma\left(\left[P, f_{2}\right]\right)\right) & 0 \\
0 & \left.f_{0}\right|_{\partial M} \circ^{\prime}\left[S,\left.f_{1}\right|_{\partial M}\right]^{\prime} \circ^{\prime}\left[S,\left.f_{2}\right|_{\partial M}\right]^{\prime}
\end{array}\right)
\end{aligned}
$$

where $\circ^{\prime}$ represents the symbol composition with respect to $\left(x^{\prime}, \xi^{\prime}\right)$. Here $\sigma\left(\left[P, f_{i}\right]\right)$ represents as usual the symbol of the operator $\left[P, f_{i}\right]$. Using the definition of Res for manifolds with boundary we have

$$
\begin{aligned}
\overline{\operatorname{Res}}( & \left.A\left(f_{0}\right)\left[A(P, S), A\left(f_{1}\right)\right]\left[A(P, S), A\left(f_{2}\right)\right]\right) \\
= & \int_{M} \int_{S^{n-1}} \operatorname{Tr}_{E}\left\{\sigma_{-n}\left(f_{0}\left[P, f_{1}\right]\left[P, f_{2}\right](x, \xi)\right)\right\} \sigma(\xi) d x \\
& +2 \pi \int_{\partial M} \int_{S^{n-2}} \operatorname{Tr}_{E^{\prime}}\left\{\sigma_{-(n-1)}\left(\operatorname{trace}\left\{f_{0}^{+} \circ^{\prime} L\left(\sigma\left(\left[P, f_{1}\right]\right), \sigma\left(\left[P, f_{2}\right]\right)\right)\right\}\left(x^{\prime}, \xi^{\prime}\right)\right)\right\} \\
& \quad+\operatorname{Tr}_{E^{\prime}}\left\{\sigma_{-(n-1)}\left(\left.f_{0}\right|_{\partial M} \circ^{\prime}\left[S,\left.f_{1}\right|_{\partial M}\right]^{\prime} \circ^{\prime}\left[S,\left.f_{2}\right|_{\partial M}\right]^{\prime}\left(x^{\prime}, \xi^{\prime}\right)\right)\right\} \sigma^{\prime}\left(\xi^{\prime}\right) d x^{\prime} .
\end{aligned}
$$

\subsection{A pair of bilinear functionals}

Mimicking the boundaryless case and following [21] we define:

\section{Definition 1.}

$$
B_{n, P}\left(f_{1}, f_{2}\right):=\int_{S^{n-1}} \operatorname{Tr}_{E}\left\{\sigma_{-n}\left(\left[P, f_{1}\right]\left[P, f_{2}\right](x, \xi)\right)\right\} \sigma(\xi),
$$

and

$$
\begin{aligned}
\partial B_{n, P, S}\left(f_{1}, f_{2}\right):=\int_{S^{n-2}} & \operatorname{Tr}_{E^{\prime}}\left\{\sigma _ { - ( n - 1 ) } \left(\operatorname{trace}\left\{L\left(\sigma\left(\left(\left[P, f_{1}\right]\right), \sigma\left(\left(\left[P, f_{2}\right]\right)\right)\right\}\left(x^{\prime}, \xi^{\prime}\right)\right)\right\}\right.\right. \\
& +\operatorname{Tr}_{E^{\prime}}\left\{\sigma_{-(n-1)}\left(\left(\left[S,\left.f_{1}\right|_{\partial M}\right]^{\prime} \circ^{\prime}\left[S,\left.f_{2}\right|_{\partial M}\right]\right)\left(x^{\prime}, \xi^{\prime}\right)\right)\right\} \sigma^{\prime}\left(\xi^{\prime}\right),
\end{aligned}
$$

for all $f_{i} \in C^{\infty}(\widetilde{M})$.

By definition, both $B_{n, P}$ and $\partial B_{n, P, S}$ are bilinear. Since $f_{0}$ is independent of $\xi$ we have

$$
\int_{S^{n-1}} \operatorname{Tr}_{E}\left\{\sigma_{-n}\left(f_{0}\left[P, f_{1}\right]\left[P, f_{2}\right](x, \xi)\right)\right\} \sigma(\xi)=f_{0} B_{n, P}\left(f_{1}, f_{2}\right) .
$$

The computations done in [19] with the symbol expansions for the case of empty boundary are also valid here. In particular we have in given local coordinates the explicit expression

$$
B_{n, P}\left(f_{1}, f_{2}\right)=\sum \frac{D_{x}^{\beta}\left(f_{1}\right) D_{x}^{\alpha^{\prime \prime}+\delta}\left(f_{2}\right)}{\alpha^{\prime} ! \alpha^{\prime \prime} ! \beta ! \delta !} \int_{S^{n-1}} \operatorname{Tr}\left\{\partial_{\xi}^{\alpha^{\prime}+\alpha^{\prime \prime}+\beta}\left(\sigma_{k-i}^{P}\right) \partial_{\xi}^{\delta}\left(D_{x}^{\alpha^{\prime}}\left(\sigma_{k-j}^{P}\right)\right)\right\} \sigma(\xi)
$$

with the sum taken over $\left|\alpha^{\prime}\right|+\left|\alpha^{\prime \prime}\right|+|\beta|+|\delta|+i+j=n+2 k,|\beta| \geq 1$, and $|\delta| \geq 1$. It shows that $B_{n, P}\left(f_{1}, f_{2}\right)$ is differential in $f_{1}$ and $f_{2}$. Evidently it is possible to obtain a similar expression for the summand in $\partial B_{n, P, S}$ corresponding to $S$ replacing $n$ by $n-1$ and $x$ by $x^{\prime}$.

In p. 25 of [8] we can read an expression for the degree $-(n-1)$ component of the operatorsymbol trace $(c)$ with $c=p^{+} o^{\prime} b$. It is given by

$$
\sigma_{-(n-1)}\left(\operatorname{trace} c\left(x^{\prime}, \xi^{\prime}\right)\right) \sim \sum_{j=0}^{\infty} \frac{i^{j}}{j !} \Pi_{\xi_{n}}^{\prime}\left\{\left.\sigma_{-n}\left(\partial_{\xi_{n}}^{j}\left[\partial_{x_{n}}^{j} p\left(x^{\prime}, 0, \xi^{\prime}, \xi_{n}\right) \circ^{\prime} b\left(x^{\prime}, \xi^{\prime}, \xi_{n}, \eta_{n}\right)\right]\right)\right|_{\eta_{n}=\xi_{n}}\right\} .
$$


Thus, since $f_{0}$ is independent of $\xi$,

$$
\begin{aligned}
\operatorname{trace} & \left\{\sigma_{-(n-1)}\left(f_{0}^{+} \circ^{\prime} L\left(\sigma\left(\left[P, f_{1}\right]\right), \sigma\left(\left[P, f_{2}\right]\right)\right)\right)\right\} \\
& =f\left(x^{\prime}, 0\right) \operatorname{trace}\left\{\sigma_{-(n-1)}\left(L\left(\sigma\left(\left[P, f_{1}\right]\right), \sigma\left(\left[P, f_{2}\right]\right)\right)\left(x^{\prime}, \xi^{\prime}\right)\right\} .\right.
\end{aligned}
$$

and it follows that

$$
\begin{aligned}
\int_{S^{n-2}} & \operatorname{Tr}\left\{\sigma_{-(n-1)}\left(\operatorname{trace}\left\{f_{0}^{+} \circ^{\prime} L\left(\sigma\left(\left[P, f_{1}\right]\right), \sigma\left(\left[P, f_{2}\right]\right)\right)\right\}\left(x^{\prime}, \xi^{\prime}\right)\right)\right\} \sigma^{\prime}\left(\xi^{\prime}\right) \\
= & f\left(x^{\prime}, 0\right) \int_{S^{n-2}} \operatorname{Tr}\left\{\sigma_{-(n-1)}\left(\operatorname{trace}\left\{L\left(\sigma\left(\left[P, f_{1}\right]\right), \sigma\left(\left[P, f_{2}\right]\right)\right)\right\}\left(x^{\prime}, \xi^{\prime}\right)\right)\right\} \sigma^{\prime}\left(\xi^{\prime}\right),
\end{aligned}
$$

for all $f_{i} \in C^{\infty}(\widetilde{M})$. In this way

$$
\begin{aligned}
\overline{\operatorname{Res}}( & \left.A\left(f_{0}\right)\left[A(P, S), A\left(f_{1}\right)\right]\left[A(P, S), A\left(f_{2}\right)\right]\right) \\
& =\int_{M} f_{0} B_{n, P}\left(f_{1}, f_{2}\right) d x+\left.2 \pi \int_{\partial M} f_{0}\right|_{\partial M} \partial B_{n, P, S}\left(\left.f_{1}\right|_{\partial M},\left.f_{2}\right|_{\partial M}\right) d x^{\prime} .
\end{aligned}
$$

Lemma 4. The functionals $B_{n, P}$ and $\partial B_{n, P, S}$ are bilinear and symmetric.

Proof. The symmetry of both $B_{n, P}$ and $\partial B_{n, P, S}$ is not evident from the expressions above. For $B_{n, P}$ it was obtained in [20] in the boundaryless case from the trace property of Res. Because it shares the same local expression both for empty and non-empty boundary we have that $B_{n, P}$ is symmetric.

For $\partial B_{n, P, S}$ we are going to exploit the linearity and the trace property of the noncommutative residue. Denote $\bar{f}=A(f)$ and $\bar{P}=A(P, S)$. Using that $\overline{f_{1}} \overline{f_{2}}=\overline{f_{2}} \overline{f_{1}}$ for all $f_{i} \in C^{\infty}(\widetilde{M})$ and the trace property of the noncommutative residue we have that all of $\overline{\operatorname{Res}}\left(\overline{f_{0}} \overline{f_{2}} \bar{P} \bar{P} \overline{f_{1}}-\overline{f_{0}} \overline{f_{1}} \bar{P} \bar{P} \overline{f_{2}}\right)$, $\overline{\operatorname{Res}}\left(\overline{f_{0}} \overline{f_{2}} \bar{P} \bar{P} \overline{f_{1}}-\overline{f_{0}} \overline{f_{1}} \bar{P} \bar{P} \overline{f_{2}}\right)$, and $\overline{\operatorname{Res}}\left(\overline{f_{0}} \bar{P} \overline{f_{2}} \bar{P} \overline{f_{1}}-\overline{f_{1}} \overline{f_{0}} \bar{P} \overline{f_{2}} \bar{P}\right)$ vanish. In this way

$$
\begin{aligned}
\overline{\operatorname{Res}}\left(\overline{f_{0}}\right. & {\left.\left[\bar{P}, \overline{f_{1}}\right]\left[\bar{P}, \overline{f_{2}}\right]-\overline{f_{0}}\left[\bar{P}, \overline{f_{2}}\right]\left[\bar{P}, \overline{f_{1}}\right]\right) } \\
= & \overline{\operatorname{Res}}\left(\overline{f_{0}} \bar{P} \overline{f_{1}} \bar{P} \overline{f_{2}}-\overline{f_{0}} \bar{P} \overline{f_{1}} \overline{f_{2}} \bar{P}-\overline{f_{0}} \overline{f_{1}} \bar{P} \bar{P} \overline{f_{2}}+\overline{f_{0}} \overline{f_{1}} \bar{P} \overline{f_{2}} \bar{P}\right. \\
& \left.-\overline{f_{0}} \bar{P} \overline{f_{2}} \bar{P} \overline{f_{1}}+\overline{f_{0}} \bar{P} \overline{f_{2}} \overline{f_{1}} \bar{P}+\overline{f_{0}} \overline{f_{2}} \bar{P} \bar{P} \overline{f_{1}}-\overline{f_{0}} \overline{f_{2}} \bar{P} \overline{f_{1}} \bar{P}\right) \\
= & \overline{\operatorname{Res}}\left(\overline{f_{0}} \bar{P} \overline{f_{1}} \bar{P} \overline{f_{2}}+\overline{f_{0}} \overline{f_{1}} \bar{P} \overline{f_{2}} \bar{P}-\overline{f_{0}} \bar{P} \overline{f_{2}} \bar{P} \overline{f_{1}}-\overline{f_{0}} \overline{f_{2}} \bar{P} \overline{f_{1}} \bar{P}\right)=0 .
\end{aligned}
$$

Hence

$$
\begin{aligned}
\int_{M} & f_{0} B_{n, P}\left(f_{1}, f_{2}\right)+\left.2 \pi \int_{\partial M} f_{0}\right|_{\partial M} \partial B_{n, P, S}\left(f_{1}, f_{2}\right) \\
& =\int_{M} f_{0} B_{n, P}\left(f_{2}, f_{1}\right)+\left.2 \pi \int_{\partial M} f_{0}\right|_{\partial M} \partial B_{n, P, S}\left(f_{2}, f_{1}\right), \quad \forall f_{i} \in C^{\infty}(\widetilde{M}) .
\end{aligned}
$$

Since $B_{n, P}\left(f_{1}, f_{2}\right)$ is symmetric

$$
\left.\int_{\partial M} f_{0}\right|_{\partial M} \bar{B}_{S}\left(f_{1}, f_{2}\right)=\left.\int_{\partial M} f_{0}\right|_{\partial M} \bar{B}_{S}\left(f_{2}, f_{1}\right), \quad \forall f_{0} \in C^{\infty}(\widetilde{M})
$$

and the result follows from the arbitrariness of $f_{0}$.

Lemma 5. $\partial B_{n, P, S}\left(f_{1}, f_{2}\right)$ is differential on $f_{1}$ and $f_{2}$. 
Proof. We denote, to simplify the notation, $P_{1}=\left[P, f_{1}\right]$ and $P_{2}=\left[P, f_{2}\right]$ with symbols $p_{1}$ and $p_{2}$ respectively. In p. 27 of [8] we can read the following

$$
\begin{aligned}
\operatorname{trace} & \left\{L\left(p_{1}, p_{2}\right)\right\}\left(x^{\prime}, \xi^{\prime}\right) \\
& =\sum_{j, k=0}^{\infty} \frac{(-i)^{j+k+1}}{(j+k+1) !} \Pi_{\xi_{n}}^{\prime}\left(\partial_{x_{n}}^{j} \partial_{\xi_{n}}^{k} \Pi_{\xi_{n}}^{+}\left(p_{1}\right)\left(x^{\prime}, 0, \xi^{\prime}, \xi_{n}\right) \circ^{\prime} \partial_{x_{n}}^{j+1} \partial_{\xi_{n}}^{k} \Pi_{\xi_{n}}^{+}\left(p_{2}\right)\left(x^{\prime}, 0, \xi^{\prime}, \xi_{n}\right)\right),
\end{aligned}
$$

with $\Pi_{\xi_{n}}^{+}(s)(\cdot)$ the projection of the symbol $s$ on $H^{+}$. The subscript in $\Pi^{+}$indicates the variable it is acting on. From [20] we know

$$
\sigma_{-k}([P, f])=\sum_{|\beta|=1}^{k} \frac{1}{\beta !} D_{x}^{\beta}(f) \partial_{\xi}^{\beta}\left(\sigma_{-(k-|b|)}^{P}\right)
$$

thus

$$
\begin{aligned}
\Pi_{\xi_{n}}^{+}\left(\sigma_{-k}([P, f])\right)\left(x^{\prime}, 0, \xi^{\prime}, \xi_{n}\right) & =\sum_{|\beta|=1}^{k} \frac{1}{\beta !} \Pi_{\xi_{n}}^{+}\left(D_{x}^{\beta}(f) \partial_{\xi}^{\beta}\left(\sigma_{-(k-|b|)}^{P}\right)\right)\left(x^{\prime}, 0, \xi^{\prime}, \xi_{n}\right) \\
& =\sum_{|\beta|=1}^{k} \frac{1}{\beta !} D_{x}^{\beta}(f)\left(x^{\prime}, 0\right) \Pi_{\xi_{n}}^{+}\left(\partial_{\xi}^{\beta}\left(\sigma_{-(k-|b|)}^{P}\right)\right)\left(x^{\prime}, 0, \xi^{\prime}, \xi_{n}\right) .
\end{aligned}
$$

Since any $\partial_{x_{n}}^{j} f$ factors out of $\Pi_{\xi_{n}}^{\prime}$ we conclude the result.

\subsection{Conformal invariance of $B_{n, P}$ and $\partial B_{n, P, S}$}

If we further assume Riemannian structures $(M, g)$ and $(\widetilde{M}, \widetilde{g})$ such that $g$ coincides with $\widetilde{g}$ restricted to $M$ then, a conformal rescaling of $g$ corresponds to a conformal rescaling of $\widetilde{g}$ (by an appropriate extension of the conformal factor) and a conformal rescaling of $\widetilde{g}$ can be restricted to a conformal rescaling of $g$. We obtain

Lemma 6. Assume that $P$ and $S$ are such that $\left[P, f_{1}\right]\left[P, f_{2}\right]$ and $\left[S,\left.f_{1}\right|_{\partial M}\right]\left[S,\left.f_{2}\right|_{\partial M}\right]$ are conformally invariant for all $f_{i} \in C^{\infty}(\widetilde{M})$. Then

$$
\widehat{B_{n, P}}\left(f_{1}, f_{2}\right)(x)=e^{-2 n \eta(x)} B_{n, P}\left(f_{1}, f_{2}\right)(x)
$$

and

$$
\widehat{\partial B_{n, P, S}}\left(f_{1}, f_{2}\right)\left(x^{\prime}\right)=e^{-2(n-1) \eta\left(x^{\prime}, 0\right)} \partial B_{n, P, S}\left(f_{1}, f_{2}\right)\left(x^{\prime}\right) .
$$

Proof. We want to exploit the independence of $\overline{\text { Res }}$ of local representations. We have

$$
\begin{aligned}
\overline{\operatorname{Res}} & \left.A\left(f_{0}\right)\left[A(P, S), A\left(f_{1}\right)\right]\left[A(P, S), A\left(f_{2}\right)\right]\right) \\
& =\int_{M} f_{0} B_{n, P}\left(f_{1}, f_{2}\right) d x+\left.2 \pi \int_{\partial M} f_{0}\right|_{\partial M} \partial B_{n, P, S}\left(\left.f_{1}\right|_{\partial M},\left.f_{2}\right|_{\partial M}\right) d x^{\prime} \\
& =\int_{M} f_{0} \widehat{B_{n, P}}\left(f_{1}, f_{2}\right) \widehat{d x}+\left.2 \pi \int_{\partial M} f_{0}\right|_{\partial M} \partial \widehat{B_{n, P}, S}\left(\left.f_{1}\right|_{\partial M},\left.f_{2}\right|_{\partial M}\right) \widehat{d x^{\prime}} \\
& =\int_{M} f_{0} e^{-2 n \eta} \widehat{B_{n, P}}\left(f_{1}, f_{2}\right) d x+\left.2 \pi \int_{\partial M} f_{0}\right|_{\partial M} e^{-\left.2(n-1) \eta\right|_{\partial M}} \widehat{\partial B_{n, P, S}}\left(\left.f_{1}\right|_{\partial M},\left.f_{2}\right|_{\partial M}\right) d x^{\prime},
\end{aligned}
$$

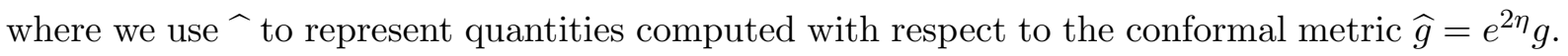
In particular

$$
\int_{M} f_{0}(x) B_{n, P}\left(f_{1}, f_{2}\right)(x) d x=\int_{M} f_{0}(x) e^{-2 n \eta(x)} \widehat{B_{n, P}}\left(f_{1}, f_{2}\right)(x) d x
$$


for all $f_{i} \in C^{\infty}(\widetilde{M})$ with $\left.f_{0}\right|_{\partial M}=0$. Thus $B_{n, P}\left(f_{1}, f_{2}\right)(x)=e^{-2 n \eta(x)} \widehat{B_{n, P}}\left(f_{1}, f_{2}\right)(x)$ for all $x \in M$. It follows that

$$
\int_{\partial M} f_{0}\left(x^{\prime}, 0\right) \widehat{B_{n, P}}\left(f_{1}, f_{2}\right)\left(x^{\prime}\right) \widehat{d x^{\prime}}=\int_{\partial M} f_{0}\left(x^{\prime}, 0\right) e^{-2 n \eta\left(x^{\prime}, 0\right)} \widehat{B_{n, P}}\left(f_{1}, f_{2}\right)\left(x^{\prime}\right) d x^{\prime}
$$

for all $f_{i} \in C^{\infty}(\widetilde{M})$. The result follows from the arbitrariness of $f_{0}$.

Remark 2. Note how the same reasoning in the proof above can be used to show the uniqueness of $B_{n, P}$ and $\partial B_{n, P, S}$ satisfying (4).

Summarizing this section we have

Theorem 4. Let $M$ be a compact manifold of dimension $n$ and with boundary $\partial M$. Assume that $M$ is embedded in a compact oriented manifold $\widetilde{M}$ without boundary. Further assume Riemannian structures $(\widetilde{M}, \widetilde{g})$ and $(M, g)$ such that $g$ coincides with $\widetilde{g}$ restricted to $M$. Let $P$ be a pseudodifferential operator acting on a vector bundle $E$ over $\widetilde{M}$ having the transmission property up to $\partial M$, let $S$ be a pseudodifferential operator acting on a vector bundle $E^{\prime}$ over $\partial M$, such that $\left[P, f_{1}\right]\left[P, f_{2}\right]$ and $\left[S,\left.f_{1}\right|_{\partial M}\right]\left[S,\left.f_{2}\right|_{\partial M}\right]$ are conformally invariant for all $f_{i} \in C^{\infty}(\widetilde{M})$. Then $B_{n, P}$ and $\partial B_{n, P, S}$ given in Definition 1 are conformally invariant in the sense of Lemma 6 . Furthermore, both $B_{n, P}$ and $\partial B_{n, P, S}$ are symmetric, bilinear differential functionals uniquely determined by the relation (4).

\section{On even-dimensional manifolds with boundary}

Up to this point, we have a generalization of Theorem 1 in [20] to manifolds with boundary in the setting described above. Next, we want to state a generalization of Theorem 2 in [20] to this context. In order to do it, we consider the Fredholm module $(\mathcal{H}, F)$ now associated to the even-dimensional manifold without boundary $\widetilde{M}$.

\subsection{The symbol of $F$ and the transmission property}

If $\omega=d \beta+\delta \beta^{\prime} \in d\left(\Omega^{n / 2-1}(\widetilde{M})\right) \oplus \delta\left(\Omega^{n / 2+1}(\widetilde{M})\right)$ then

$$
\Delta F_{0}\left(d \beta+\delta \beta^{\prime}\right)=\Delta\left(d \beta-\delta \beta^{\prime}\right)=d \delta d \beta-\delta d \delta \beta^{\prime}=F_{0}\left(d(\delta d \beta)+\delta\left(d \delta \beta^{\prime}\right)\right)=F_{0} \Delta\left(d \beta+\delta \beta^{\prime}\right) .
$$

It follows

Lemma 7. For an oriented compact manifold without boundary $\widetilde{M}$ and of even dimension $n$, the relation $F_{0} \Delta=\Delta F_{0}=d \delta-\delta d$ holds on $d\left(\Omega^{n / 2-1}(\widetilde{M})\right) \oplus \delta\left(\Omega^{n / 2+1}(\widetilde{M})\right)$.

To be able to use a given pseudodifferential operator in the machinery of the noncommutative residue for manifolds with boundary, it is essential for the operator to enjoy the transmission property up to the boundary of $M$.

Because we are interested in $F$ acting on the orthogonal complement of the harmonic forms on $\widetilde{M}$, we abuse of the notation and use freely $F$ for $F_{0}$. From the relation $\Delta F=d \delta-\delta d$ and the formula for the total symbol of the product of pseudodifferential operators we can compute the symbol expansion of $F$. First we note that $F$ is a pseudodifferential operator of order 0 .

We know $\sigma(\Delta F)=\sigma(d \delta-\delta d)$, thus the formula for the total symbol of the product of two pseudodifferential operators implies

$$
\begin{aligned}
\sigma_{2}^{d \delta-\delta d}+\sigma_{1}^{d \delta-\delta d}+\sigma_{0}^{d \delta-\delta d} & =\sigma(d \delta-\delta d)=\sigma(\Delta F) \sim \sum \frac{1}{\alpha !} \partial_{\xi}^{\alpha} \sigma(\Delta) D_{x}^{\alpha}(\sigma(F)) \\
& \sim \sum \frac{1}{\alpha !} \partial_{\xi}^{\alpha}\left(\sigma_{2}^{\Delta}+\sigma_{1}^{\Delta}+\sigma_{0}^{\Delta}\right) D_{x}^{\alpha}\left(\sigma_{0}^{F}+\sigma_{-1}^{F}+\sigma_{-2}^{F}+\cdots\right) .
\end{aligned}
$$

Expanding the right hand side into sum of terms with the same homogeneity we conclude: 
Lemma 8. In any given system of local charts, we can express the total symbol of $F, \sigma(F) \sim$ $\sigma_{0}^{F}+\sigma_{-1}^{F}+\cdots$ in a recursive way by the formulae:

$$
\begin{aligned}
\sigma_{0}^{F}= & \left(\sigma_{2}^{\Delta}\right)^{-1} \sigma_{2}^{d \delta-\delta d}, \quad \sigma_{-1}^{F}=\left(\sigma_{2}^{\Delta}\right)^{-1}\left(\sigma_{1}^{d \delta-\delta d}-\sigma_{1}^{\Delta} \sigma_{0}^{F}-\sum_{|\alpha|=1} \partial_{\xi}^{\alpha}\left(\sigma_{2}^{\Delta}\right) D_{x}^{\alpha}\left(\sigma_{0}^{F}\right)\right) \\
\sigma_{-2}^{F}= & \left(\sigma_{2}^{\Delta}\right)^{-1}\left(\sigma_{0}^{d \delta-\delta d}-\sigma_{1}^{\Delta} \sigma_{-1}^{F}-\sigma_{0}^{\Delta} \sigma_{0}^{F}\right. \\
& \left.-\sum_{|\alpha|=1}\left(\partial_{\xi}^{\alpha}\left(\sigma_{2}^{\Delta}\right) D_{x}^{\alpha}\left(\sigma_{-1}^{F}\right)+\partial_{\xi}^{\alpha}\left(\sigma_{1}^{\Delta}\right) D_{x}^{\alpha}\left(\sigma_{0}^{F}\right)\right)-\sum_{|\alpha|=2} \frac{1}{\alpha !} \partial_{\xi}^{\alpha}\left(\sigma_{2}^{\Delta}\right) D_{x}^{\alpha}\left(\sigma_{0}^{F}\right)\right) \\
\sigma_{-r}^{F}= & -\left(\sigma_{2}^{\Delta}\right)^{-1}\left(\sigma_{1}^{\Delta} \sigma_{-r+1}^{F}+\sigma_{0}^{\Delta} \sigma_{-r+2}^{F}+\sum_{|\alpha|=1} \partial_{\xi}^{\alpha}\left(\sigma_{2}^{\Delta}\right) D_{x}^{\alpha}\left(\sigma_{-r+1}^{F}\right)\right. \\
& \left.+\sum_{|\alpha|=1} \partial_{\xi}^{\alpha}\left(\sigma_{1}^{\Delta}\right) D_{x}^{\alpha}\left(\sigma_{-r+2}^{F}\right)+\sum_{|\alpha|=2} \frac{1}{\alpha !} \partial_{\xi}^{\alpha}\left(\sigma_{2}^{\Delta}\right) D_{x}^{\alpha}\left(\sigma_{-r+2}^{F}\right)\right)
\end{aligned}
$$

for every $r \geq 3$.

Lemma 2.4 of [17] states that all symbols which are polynomial in $\xi$ have the transmission property. Thus both $\Delta$ and $d \delta-\delta d$ have the transmission property. Proposition 2.7 in the same reference states that if two operators satisfy the transmission property then their products, all their derivatives, and their parametrizes satisfy the transmission property as well. Furthermore, the same result also states that it is enough to check that each homogeneous component of the symbol expansion has the transmission property to conclude that the full symbol has the transmission property.

By Lemma 8, each homogeneous component $\sigma_{-k}^{F}$ in the symbol expansion of $F$ is given in terms of derivatives of the homogeneous components of $\Delta, d \delta-\delta d, \sigma_{0}^{F}, \ldots, \sigma_{-k+1}^{F}$, and $\sigma_{2}(\Delta)^{-1}$. By Lemma 2.4 and Proposition 2.7 of [17] it follows that

Lemma 9. The operator $F$ satisfies the transmission property.

\section{$5.2 \overline{\operatorname{Res}}\left(A\left(f_{0}\right)\left[A(F, 0), A\left(f_{1}\right)\right]\left[A(F, 0), A\left(f_{2}\right)\right]\right)$}

For $F$ given in (2) now for the manifold $\widetilde{M}$, and for $f \in C^{\infty}(\widetilde{M})$ we let $A(F, 0)$ and $A(f)$ be the elements in Boutet de Monvel's algebra of diagonal elements given by

$$
\bar{F}=A(F, 0)=\left(\begin{array}{cc}
r^{M} F e^{M}+0 & 0 \\
0 & 0
\end{array}\right), \quad \bar{f}=A(f)=\left(\begin{array}{cc}
r^{M} f e^{M}+0 & 0 \\
0 & \left.f\right|_{\partial M}
\end{array}\right) .
$$

Since $L(f, \sigma(F))=0=L(\sigma(F), f)$, it follows that

$$
\overline{f_{0}}\left[\bar{F}, \overline{\left.f_{1}\right]}\right]\left[\bar{F}, \overline{f_{2}}\right]=\left(\begin{array}{cc}
r^{M} f_{0}\left[F, f_{1}\right]\left[F, f_{2}\right] e^{M}+f_{0}^{+} \circ^{\prime} L\left(\sigma\left(\left[F, f_{1}\right]\right), \sigma\left(\left[F, f_{2}\right]\right)\right) & 0 \\
0 & 0
\end{array}\right),
$$

where $o^{\prime}$ represents the symbol composition with respect to $\left(x^{\prime}, \xi^{\prime}\right)$. As before we define:

\section{Definition 2.}

$$
B_{n}\left(f_{1}, f_{2}\right):=\int_{S^{n-1}} \operatorname{Tr}\left\{\sigma_{-n}\left(\left[F, f_{1}\right]\left[F, f_{2}\right](x, \xi)\right)\right\} \sigma(\xi),
$$

and

$$
\partial B_{n}\left(f_{1}, f_{2}\right)=\int_{S^{n-2}} \operatorname{Tr} \sigma_{-(n-1)}\left\{\left(\operatorname{trace}\left\{L\left(\sigma\left(\left[F, f_{1}\right]\right), \sigma\left(\left[F, f_{2}\right]\right)\right)\left(x^{\prime}, \xi^{\prime}\right)\right\}\right)\right\} \sigma^{\prime}\left(\xi^{\prime}\right),
$$

for all $f_{i} \in C^{\infty}(\widetilde{M})$. 
As in Section 4

Theorem 5. Both differential functionals $B_{n}$ and $\partial B_{n}$ are bilinear, symmetric, conformal invariant in the sense

$$
\widehat{B_{n}}\left(f_{1}, f_{2}\right)(x)=e^{-2 n \eta(x)} B_{n}\left(f_{1}, f_{2}\right)(x)
$$

and

$$
\left.\partial B_{n} \widehat{\left(f_{1}, f_{2}\right.}\right)\left(x^{\prime}\right)=e^{-2(n-1) \eta\left(x^{\prime}, 0\right)} \partial B_{n}\left(f_{1}, f_{2}\right)\left(x^{\prime}\right)
$$

for a conformal change of the metric $\widehat{g}=e^{2 \eta} g$, and are uniquely determined by the relation

$$
\begin{aligned}
\overline{\operatorname{Res}} & \left.A\left(f_{0}\right)\left[A(F, 0), A\left(f_{1}\right)\right]\left[A(F, 0), A\left(f_{2}\right)\right]\right) \\
& =\int_{M} f_{0} B_{n, P}\left(f_{1}, f_{2}\right) d x+\left.2 \pi \int_{\partial M} f_{0}\right|_{\partial M} \partial B_{n, P, S}\left(f_{1}, f_{2}\right) d x^{\prime} .
\end{aligned}
$$

Remark 3. Even though both bilinear functionals $B_{n}$ and $\partial B_{n}$ are acting on $C^{\infty}(\widetilde{M})$, they depend on the particular embedding of the compact manifold $M$ into $\widetilde{M}$, and thus, they can be defined on $C^{\infty}(M)$ by considering an extension of $f \in C^{\infty}(M)$ to $C^{\infty}(\widetilde{M})$.

Remark 4. In case $M$ is odd dimensional, all results from the first part of these notes are valid on the compact even dimensional manifold without boundary $\partial M$. In this way, we can consider the commutative algebra $\mathcal{A}=C^{\infty}(\partial M)$ and the Fredholm module associated to the manifold $\partial M$. For $F$ given in (2) on the manifold $\partial M$, we could look at

$$
A(P, F)=\left(\begin{array}{cc}
P & 0 \\
0 & F
\end{array}\right)
$$

and try to study $\operatorname{Res}\left(A\left(f_{0}\right)\left[A(P, F), A\left(f_{1}\right)\right]\left[A(P, F), A\left(f_{2}\right)\right]\right)$ for functions $f_{i} \in C^{\infty}(\widetilde{M})$. The trivial choice $P=0$ will produce $B_{n, P}=0$ and $\partial B_{n, 0, F}=B_{n-1}$. It is an open problem to search for a companion $P$ for $F$ that will produce more interesting results in the odd dimensional case.

\section{Acknowledgements}

This research is supported by Vicerrectoría de Investigación de la Universidad de Costa Rica and Centro de Investigaciones Matemáticas y Meta-matemáticas. The material extends a talk presented in May 2007 at the Midwest Geometry Conference held at the University of Iowa in honor of Thomas P. Branson.

The referees' suggestions improved to a great extent the presentation of this material. One of the referees pointed the author towards [11] which provided a clearer understanding of Boutet de Monvel's calculus. In particular, the formulae used for $L(p, q)$ resulted in a significant simplification of the treatment of the subject.

\section{References}

[1] Adler M., On a trace functional for formal pseudodifferential operators and the symplectic structure of the Korteweg-de Vries type equations, Invent. Math. 50 (1979), 219-248.

[2] Bär C., Conformal structures in noncommutative geometry, J. Noncommut. Geom. 1 (2007), 385-395, arXiv:0704.2119.

[3] Boutet de Monvel L., Boundary problems for pseudo-differential operators, Acta Math. 126 (1971), 11-51.

[4] Connes A., The action functional in noncommutative-geometry, Comm. Math. Phys. 117 (1988), 673-683. 
[5] Connes A., Quantized calculus and applications, in Proceedings of the XIth International Congress of Mathematical Physics, International Press, Cambridge, MA, 1995, 15-36.

[6] Connes A., Noncommutative geometry, Academic Press, London and San Diego, 1994.

[7] Connes A., Sullivan D., Teleman N., Quasiconformal mappings, operators on Hilbert space, and local formulae for characteristic classes, Topology 33 (1994), 663-681.

[8] Fedosov B.V., Golse F., Leichtnam E., Schrohe E., The noncommutative residue for manifolds with boundary, J. Funct. Anal. 142 (1996), 1-31.

[9] Gracia-Bondía J.M., Várilly J.C., Figueroa H., Elements of noncommutative geometry, Birkhäuser Advanced Texts, Birkhäuser, Boston, 2001.

[10] Graham R., Jenne R., Mason L., Sparling G., Conformally invariant powers of the Laplacian. I. Existence, J. London Math. Soc. (2) 46 (1992), 557-565.

[11] Grubb G., Singular Green operators and their spectral asymptotics, Duke Math. J. 51 (1984), 477-528.

[12] Guillemin V.W., A new proof of Weyl's formula on the asymptotic distribution of eigenvalues, Adv. Math. 55 (1985), 131-160.

[13] Manin Yu.I., Algebraic aspects of nonlinear differential equations, J. Sov. Math. 11 (1979), 1-22.

[14] Paneitz S., A quadratic conformally covariant differential operator for arbitrary pseudo-Riemannian manifolds, Preprint, 1983.

[15] Polyakov A., Quantum geometry of bosonic strings, Phys. Lett. B 103 (1981), 207-210.

Polyakov A., Quantum geometry of fermionic strings, Phys. Lett. B 103 (1981), 211-213.

[16] Schrohe E., Noncommutative residue, Dixmier's traces, and heat trace expansions on manifolds with boundary, Contemp. Math. 242 (1999), 161-186, math.AP/9911053.

[17] Schrohe E., A short introduction to Boutet de Monvel's calculus, in Approaches to Singular Analysis, Editors J. Gil, D. Grieser and M. Lesch, Birkhäuser, Basel, 2001, 85-116.

[18] Ugalde W.J., Differential forms canonically associated to even-dimensional compact conformal manifolds, in Clifford Algebras. Applications to Mathematics, Physics, and Engineering, Editor R. Ablamowicz, Progress in Mathematical Physics, Vol. 34, Birkhäuser, Boston, 2004, 211-225, math.DG/0211240.

[19] Ugalde W.J., A construction of critical GJMS operators using Wodzicki's residue, Comm. Math. Phys. 261 (2006), 771-788, math.DG/0403392.

[20] Ugalde W.J., Differential forms and the Wodzicki residue, math.DG/0211361.

[21] Wang Y., Differential forms and the Wodzicki residue for manifolds with boundary, J. Geom. Phys. 56 (2006), 731-753, math.DG/0609062.

[22] Wang Y., Differential forms and the noncommutative residue for manifolds with boundary in the non-product case, Lett. Math. Phys. 77 (2006), 41-51, math.DG/0609060.

[23] Wodzicki M., Local invariants of spectral asymmetry, Invent. Math. 75 (1984), 143-178. 